\title{
SELEÇÃO DE MÉTODO ESTATÍSTICO PARA MODELOS DE ESTIMATIVA DA QUALIDADE FÍSICA DE SOLOS ARGILOSOS
}

\author{
Adriana Aparecida Ribon ${ }^{1}$; João Tavares Filho²; Kathleen Lourenço Fernandes ${ }^{1}$; Victor Talles \\ Lourenceti Hermógenes ${ }^{1}$ \\ ${ }^{1}$ Universidade Estadual de Goiás - UEG, e-mail: adriana.ribon@ueg.br, kathleen_agro@hotmail.com, \\ victor_lourenceti@hotmail.com \\ ${ }^{2}$ Universidade Estadual de Londrina - UEL, e-mail: tavares@uel.br
}

\section{RESUMO}

Foram selecionados três métodos estatísticos ("Stepwise, Backward" e "Forward") para a escolha do melhor na obtenção de modelos para estimativa da qualidade física de um Latossolo Vermelho Eutroférrico sob cultura perene e um Latossolo Vermelho Distroférrico sob plantio direto. O método "Stepwise" foi o mais adequado na obtenção dos modelos finais para estimativa da qualidade física do Latossolo Vermelho Eutroférrico sob cultura perene. Os três procedimentos ("Stepwise, Forward" e "Backward") foram adequados na obtenção dos modelos finais para a estimativa da qualidade física do Latossolo Vermelho Distroférrico sob plantio direto.

Palavras-chave: Backward, forward, latossolos, stepwise

\section{SELECTION OF STATISTICAL MODELS FOR ESTIMATING PHYSICAL QUALITY OF CLAY SOILS}

\begin{abstract}
Three statistical methods (Stepwise, Backward and Forward) were selected for choosing the best procedure in obtaining models to estimate the physical quality of an Oxisoil under perennial crop and an Oxisol under no-tillage. The stepwise method was suitable for obtaining the final models for estimating the physical quality of Oxisoil under perennial crop. The three procedures (Stepwise, Forward and Backward) were adequate to obtain the final models to estimate the physical quality of Red Hapludox tillage system.
\end{abstract}

Keywords: Backward, forward, oxisols, stepwise

\section{INTRODUÇÃO}

A análise de regressão é uma técnica amplamente utilizada nas diversas áreas da ciência a fim de estudar a correlação entre diferentes variáveis que interagem entre si. $\mathrm{Na}$ ciência do solo, a análise de regressão possibilita o entendimento dos atributos do solo e suas interações facilitando as técnicas de manejo e a escolha das mesmas. De acordo com Stefanoski et al. (2013), técnicas que visem avaliar a qualidade de um solo de forma simples e confiável ainda são objeto de investigações, e resultados sistemáticos deste monitoramento representam papel central em estudos sobre qualidade do solo.

Assim, diversos trabalhos visam o estudo das interações entre atributos físicos do solo para estimativa da qualidade do solo, através de modelos estatísticos que 
envolvam atributos físicos facilmente mensuráveis. De acordo com Singer \& Ewing (2000) a porosidade total, distribuição e tamanho dos poros, distribuição do tamanho das partículas, densidade do solo e resistência do solo à penetração das raízes estão entre os atributos mais utilizados como indicadores de qualidade física do solo.

Albuquerque et al. (2001) correlacionaram os atributos físicos do solo ao sistema de manejo. Conforme os autores, os macroporos estão mais sujeitos à alteração em função do manejo que os microporos em LATOSSOLOS, sendo a maior ocorrência de macroporos em sistema de plantio convencional e menor em sistemas de plantio direto. Estes macroporos influenciam significativamente na porosidade total. Lanzanova et al. (2007) explicam que há interações entre a compactação e o aumento da densidade do solo e resistência do solo à penetração em condições de baixa umidade. As hipóteses existentes é que há uma relação inversa entre a intensidade de uso do solo e uma boa qualidade do mesmo (ARAÚJO et al., 2007).

Desta forma, o monitoramento dos atributos físicos do solo é de extrema importância para se conhecer a qualidade real do solo e capacidade que este terá para a produção. A qualidade do solo é dada como as condições ótimas que este apresenta para seu funcionamento e sua qualidade física descreve como o solo permite a infiltração, retenção e disponibilidade de água às plantas, a forma como responde ao manejo e resiste a degradação, permite as trocas de calor e de gases com a atmosfera e raízes e permite o crescimento das raízes (AGUIAR, 2008). Araújo et al. (2007) explicam que a mensuração destes atributos deve ser por meio de técnicas que monitoram o estado do solo ao longo do tempo, como linhas de modelagem gráfica ou matemática que permitem o monitoramento da propriedade agrícola auxiliando na adoção de práticas de manejo e conservação do solo e da água procurando manter seus atributos de modo a aumentar a capacidade do solo em sustentar sua produtividade.

De acordo com Hocking (1976), existem vários métodos para avaliação de apenas um pequeno número de subconjuntos que possibilitam adicionar ou excluir variáveis uma por vez, de acordo com um critério específico. Porém, as soluções encontradas são muitas vezes iguais (DRAPER \& SMITH, 1966). Os principais procedimentos utilizados são: todas as equações possíveis (AR), "backward elimination", "forward selection" e "stepwise".

Entretanto, a crítica mais comum, de acordo com Efroymson (1960), é que nem "backward, forward" ou "stepwise" assegurarão, com as óbvias exceções, que o "melhor subconjunto" de um determinado conjunto será revelado. Alguns recomendam tanto um quanto os outros para que haja uma concordância no resultado final obtido. Para a seleção das equações que melhor explicam o efeito das variáveis independentes em estudo dentro de um determinado procedimento escolhido são utilizadas diversas funções, sendo as mais usadas:

- Comparação entre a soma de quadrados dos desvios da regressão: quanto menor a soma de quadrados dos desvios, melhor a equação;

- O quadrado médio do resíduo: a melhor equação é a que apresenta menor quadrado médio do resíduo;

- Coeficiente de determinação $\left(\mathrm{R}^{2}\right)$ : a melhor equação é a que apresenta o valor de $\mathrm{R}^{2}$ mais elevado;

- Estatística Cp: A melhor estimativa é aquela expressa pela regressão: $\mathrm{Cp}=\mathrm{K}=1$.

Dentre as funções utilizadas, a escolha pelo coeficiente de determinação é muito empregada (DRAPER \& SMITH, 1966). 
O procedimento "stepwise" tem tido maior aceitação nos estudos dentro da área da ciência do solo e vem sendo altamente empregado no estudo das relações entre parâmetros de solos tropicais. Dessa forma, Dias et al. (1997), verificaram que "stepwise" é um bom procedimento para verificar a relação entre a produtividade, clima, solos e variedades de cana de açúcar, na região Noroeste do estado de São Paulo. O procedimento "stepwise" também foi utilizado por Ribon et al. (2001) na obtenção de regressões múltiplas para explicar a relação entre o desenvolvimento da seringueira em Latossolo e Argissolo, verificando ser esta opção satisfatória, salientando que as equações obtidas devem ser usadas dentro dos limites dos valores das variáveis do solo para os quais foi calculada. Recentemente, vários estudos da qualidade física dos solos vem empregando o procedimento "stepwise" (FIDALSKI \& TORMENA, 2007; TAVARES FILHO et al., 2012; STONE et al., 2013).

Desta forma, o presente trabalho tem como objetivo a seleção do melhor método e modelos finais obtidos no estudo de relações entre parâmetros físicos de solos argilosos nas regiões oeste e norte do Paraná.

\section{MATERIAL E MÉTODOS}

Os dados utilizados no presente trabalho foram provenientes de dois experimentos realizados em duas áreas experimentais, sendo uma delas constituída por um Latossolo Vermelho Eutroférrico (LVe) (teor de argila na camada de 0-0,6m variando de 730 a $800 \mathrm{~g} \mathrm{~kg}^{-1}$ ) em uma área agrícola de 1 ha, na região norte do Estado do Paraná com características climáticas Cfa-subtropical úmido, segundo classificação de Köpen. O outro experimento foi conduzido em um Latossolo Vermelho Distroférrico típico (LVd) (EMBRAPA, 2013), com teor de argila na camada de 0$0,6 \mathrm{~m}$ variando entre 500 e $850 \mathrm{~g} \mathrm{~kg}^{-1}$ em uma área agrícola de 1ha, na região oeste do Paraná sendo cultivada há sete anos em sistema plantio direto, sem rotação de culturas, de forma sucessiva a cultura da aveia preta no inverno e a do milho no verão. Na safra de verão 2000/2003, implantou-se pela primeira vez após sete anos a cultura da soja.

Os dados utilizados são provenientes de determinações físicas obtidas nas áreas experimentais, considerando os seguintes atributos físicos dos solos: resistência do solo à penetração (RP) como variável dependente (Y) e densidade do solo (DS), umidade gravimétrica (UG), matéria orgânica (MO), porosidade total (PT) e conteúdo de argila (ARG), como variáveis independentes (X1, X2, X3, X4 e X5, respectivamente)

Os modelos foram gerados de acordo com os procedimentos "backward, forward" e "stepwise" e a seleção do melhor modelo foi através do coeficiente de determinação e do valor de $\mathrm{F}$ pré estabelecido ao nível de 0,05 de probabilidade.

A eliminação "backward" consiste na obtenção de um modelo, onde inicia-se pela equação na qual todas as variáveis estão inclusas e estas são eliminadas uma por vez a cada passo à partir de um nível de significância pré-estabelecido, ou seja, as variáveis eliminadas vão apresentar o nível de significância mais elevado, dentre as variáveis incluídas, até que o nível de significância do valor de $\mathrm{F}$ de todas as variáveis seja menor que o do modelo pré estabelecido. Para esse procedimento, todas as variáveis participantes do modelo são significativas ao nível 0,10 de probabilidade.

$\mathrm{Na}$ seleção "forward" o procedimento se inicia com nenhuma outra variável na equação e se adiciona uma por vez, iniciando-se pela variável que promover um decréscimo mais significativo na soma de quadrados do resíduo e que apresente um nível de significância do valor de $\mathrm{F}$, menor que um valor pré-estabelecido, até que todas 
as variáveis com nível de significância do valor de $\mathrm{F}$ menor do que esse valor estejam dentro do modelo. Para esse procedimento nenhuma outra variável com nível de significância maior que $0,05 \quad(5 \%)$ de probabilidade será inclusa.

No procedimento "stepwise", o procedimento torna-se completo, quando todas as variáveis incluídas no modelo apresentam nível de significância do valor de $\mathrm{F}$ menor que o valor pré-estabelecido para eliminação de variáveis, e todas as variáveis fora do modelo têm nível de significância do valor $\mathrm{F}$ maior que o valor pré-estabelecido para inclusão de variáveis.

\section{RESULTADOS E DISCUSSÃO}

Os resumos dos métodos estatísticos "backward, forward" e "stepwise", provindos das análises do Latossolo
Vermelho Eutroférrico sob cultura perene encontram-se expressos (Tabelas 1 a 3 ).

No procedimento "backward elimination" (Tabela 1), o valor do coeficiente de determinação quando todas as variáveis são consideradas (passo 0) é de 0,6015 . No passo 1 , a variável porosidade total (PT) é removida. De acordo com esse procedimento, todas as variáveis estão incluídas e, a partir dele, eliminam-se uma a uma as variáveis que em cada passo, apresentam o nível de significância de $\mathrm{F}$ mais elevado, dentre as variáveis incluídas, até que o nível de significância do valor de $\mathrm{F}$ de todas as variáveis do modelo seja menor que um valor pré-estabelecido (HOCKING, 1976).

Deste modo, a variável PT foi a única que apresentou um nível de significância acima de $10 \%$ de probabilidade, tendo dessa forma sido excluída.

Tabela 1. Procedimento "Backward elimination" para variável dependente (Y) resistência do solo à penetração (RP) de dados provindos do Latossolo Vermelho Eutroférrico sob cultura perene.

\begin{tabular}{llrl}
\hline Passo 0 $\left(\mathrm{R}^{2}=0,60\right)$ & Todas as variáveis consideradas & & \\
Variáveis & Estimativa de parâmetros & $\mathrm{F}$ & Prob>F \\
\hline Intercepto & 2,1169 & 3,73 & 0,0543 \\
DS & $-6,7590$ & 61,21 & 0,0001 \\
UG & 5,9885 & 141,68 & 0,0001 \\
MO & 0,0123 & 3,32 & 0,0693 \\
PT & $-1,1857$ & 1,94 & 0,1647 \\
ARG & $-0,0025$ & 6,09 & 0,0141 \\
\hline Passo1 $\left(\mathrm{R}^{2}=0,599\right)$ & Remoção da variável PT & & \\
Intercepto & 1,5288 & 2,28 & 0,1322 \\
\hline DS & $-6,9237$ & 65,36 & 0,0001 \\
UG & 5,9071 & 139,38 & 0,0001 \\
MO & 0,0115 & 2,91 & 0,0891 \\
ARG & $-0,0024$ & 5,67 & 0,0177 \\
\hline
\end{tabular}

*Todas as variáveis participantes do modelo são significativas ao nível de $0,1000 \%$ de probabilidade. RP resistência do solo à penetração; DS - densidade do solo; UG - umidade gravimétrica; MO - matéria orgânica; PT porosidade total; ARG - argila. 
Tabela 2. Procedimento "Forward Selection" para variável dependente (Y) resistência do solo à penetração $(\mathrm{RP})$.

\begin{tabular}{|c|c|c|c|}
\hline Passo $1\left(\mathrm{R}^{2}=0,514\right)$ & Seleção da variável UG & & \\
\hline Variáveis & Estimativa de parâmetros & $\mathrm{F}$ & Prob $>F$ \\
\hline Intercepto & $-4,5490$ & 90,07 & 0,0001 \\
\hline UG & 8,3474 & 390,37 & 0,0001 \\
\hline Passo $2\left(\mathrm{R}^{2}=0,5875\right)$ & Seleção da variável DS & & \\
\hline Intercepto & 0,3054 & 0,17 & 0,6820 \\
\hline DS & $-7,0075$ & 65,01 & 0,0001 \\
\hline $\mathrm{UG}$ & 5,7584 & 130,52 & 0,0001 \\
\hline Passo3 $\left(\mathrm{R}^{2}=0,5962\right)$ & Seleção da variável ARG & & \\
\hline Intercepto & 2,0569 & 4,52 & 0,0341 \\
\hline DS & $-6,8841$ & 64,33 & 0,0001 \\
\hline UG & 5,8485 & 136,57 & 0,0001 \\
\hline $\mathrm{ARG}$ & $-0,0028$ & 7,84 & 0,0054 \\
\hline Passo4 $\left(\mathrm{R}^{2}=0,5994\right)$ & Seleção da variável MO & & \\
\hline Intercepto & 1,5288 & 2,28 & 0,1322 \\
\hline DS & $-6,9237$ & 65,36 & 0,0001 \\
\hline $\mathrm{UG}$ & 5,9071 & 139,38 & 0,0001 \\
\hline MO & 0,0115 & 2,91 & 0,0891 \\
\hline ARG & $-0,00242$ & 5,67 & 0,0177 \\
\hline $\operatorname{Passo5}\left(\mathrm{R}^{2}=0,6015\right)$ & Seleção da variável PT & & \\
\hline Intercepto & 2,1169 & 3,73 & 0,0543 \\
\hline DS & $-6,7590$ & 61,27 & 0,0001 \\
\hline $\mathrm{UG}$ & 5,9885 & 141,68 & 0,0001 \\
\hline MO & 0,0123 & 3,32 & 0,0693 \\
\hline PT & $-1,1857$ & 1,94 & 0,1647 \\
\hline ARG & $-0,0025$ & 6,09 & 0,0141 \\
\hline
\end{tabular}

*Todas as variáveis selecionadas para o modelo são significativas ao nível de 0,5000 \% de probabilidade. RP resistência do solo à penetração; DS - densidade do solo; UG - umidade gravimétrica; MO - matéria orgânica; PT porosidade total; ARG - argila. 
Tabela 3. Procedimento "Stepwise" para variável dependente (Y) resistência do solo à penetração (RP).

\begin{tabular}{llrl}
\hline Passo 1 $\left(\mathrm{R}^{2}=0,514\right)$ & Seleção da variável UG & \\
\hline Variáveis & Estimativa de parâmetros & $\mathrm{F}$ & Prob>F \\
Intercepto & $-4,5490$ & 90,07 & 0,0001 \\
UG & 8,3474 & 390,37 & 0,0001 \\
\hline Passo 2 $\left(\mathrm{R}^{2}=0,5875\right)$ & Seleção da variável DS & \\
\hline Intercepto & 0,3054 & 0,17 & 0,6820 \\
DS & $-7,0075$ & 65,01 & 0,0001 \\
UG & 5,7584 & 130,52 & 0,0001 \\
\hline Passo3 $\left(\mathrm{R}^{2}=0,5962\right)$ & Seleção da variável ARG & & \\
\hline Intercepto & 2,0569 & 4,52 & 0,0341 \\
DS & $-6,8841$ & 64,33 & 0,0001 \\
UG & 5,8485 & 136,57 & 0,0001 \\
ARG & $-0,0028$ & 7,84 & 0,0054 \\
\hline Passo4 $\left(\mathrm{R}^{2}=0,5994\right)$ & Seleção da variável MO & & \\
\hline Intercepto & 1,5288 & 2,951 & 0,0891 \\
DS & $-6,9237$ & 5,67 & 0,0177 \\
UG & 5,9071 & 139,38 & 0,0001 \\
MO & 0,0115 & 2,1322 \\
ARG & $-0,00242$ & 0,0001 \\
\hline
\end{tabular}

*Todas as variáveis selecionadas para o modelo são significativas ao nível de 0,5000 \% de probabilidade. RP resistência do solo à penetração; DS - densidade do solo; UG - umidade gravimétrica; MO - matéria orgânica; PT porosidade total; ARG - argila.

Entretanto, no modelo final obtido por este procedimento algumas variáveis, tal como a MO, não atendem ao valor da prob $<$ F ao nível de 1 e $5 \%$ de probabilidade, não sendo este método o mais adequado para esse caso, uma vez que esta variável não contribui significativamente para explicar a relação entre RP e as variáveis independentes.

No procedimento "Forward" (Tabela 2), inverso do anterior, ou seja, inicia-se com a equação que só tem b0 e vai se acrescentando à equação as variáveis, uma a uma, iniciando-se pela variável que apresente um nível de significância do valor de $\mathrm{F}$, menor que o pré estabelecido, até que todas as variáveis com nível de significância do valor de $\mathrm{F}$ menores do que esse valor estejam dentro do modelo. A primeira variável selecionada é UG (passo 1) contribuindo com $51 \%$ da variação da variável dependente. Em seguida, a variável 
selecionada é DS, promovendo um acréscimo significativo no coeficiente de determinação, elevando-o a 0,588. Nos passos 3, 4 e 5, as variáveis selecionadas são respectivamente a ARG $\left(\mathrm{R}^{2}=0,596\right), \mathrm{MO}$ $\left(\mathrm{R}^{2}=0,599\right)$ e PT $\left(\mathrm{R}^{2}=0,6015\right)$.

Observa-se no modelo final, apesar das variáveis terem sido selecionadas por esse método, que estas também não atendem ao nível de significância menor que $5 \%$ de probabilidade, dessa forma não ocorre um acréscimo significativo no coeficiente de determinação com a seleção de cada uma dessas variáveis. Portanto, o modelo ideal seria o obtido no passo 3 , com as variáveis incluídas DS, UG e ARG e um coeficiente de determinação de 0,5962 .

Em função da resistência à penetração sofrer influência de uma série de propriedades do solo, onde o teor de umidade e a densidade do solo são consideradas as mais significativas, (SILVA \& KAY, 1997; IMHOFF et al., 2000) alguns autores foram levados a propor modelos empíricos para descrever RP (ALMEIDA et al., 2008; BUSSCHER, 1990; BUSSCHER et al., 1997; DEXTER et al., 2007; LIMA et al., 2007; RIBON \& TAVARES FILHO, 2004; TO \& KAY, 2005). O modelo mais utilizado para estimar a RP é o proposto por Busscher (1990), no qual RP é obtida como uma função de densidade de volume e teor de umidade $(\theta)$, e os parâmetros de regressão "a", "b" e "c" são obtidos através de ajustes não lineares, que variam a partir de um tipo de solo para o outro. Entretanto, a relação positiva encontrada entre RP e UG pode ser explicada, de acordo com Ribon \& Tavares Filho (2004), pela pequena amplitude de umidade encontrada. A relação entre a resistência do solo à penetração e o teor de argila pode ser explicada por Sá \& Santos Júnior (2005). Estes autores citam que teor de argila afeta a coesão entre as partículas, obtendo uma estreita relação entre RP e o conteúdo de argila do solo.

O procedimento "Stepwise" (Tabela 3) mostra passo a passo a entrada ou saída das variáveis participantes do modelo traçando um caminho ótimo entre elas até a obtenção do modelo final. Observa-se que esse procedimento igualou-se ao "forward" na obtenção do modelo final, considerando apenas variáveis com prob $<\mathrm{F}$ abaixo de $5 \%$ de probabilidade para permanecer no modelo. Efroymson (1960) recomenda testar tanto um quanto os outros procedimentos, para que haja uma concordância no resultado final obtido na escolha do melhor procedimento. De acordo com Dias et al. (1997), a vantagem do "Stepwise" é que este procedimento permite uma avaliação do modelo a cada inclusão ou exclusão de uma nova variável. Entretanto, para os dados avaliados não houve exclusão de variáveis, portanto não houve diferença entre ambos os procedimentos no caminho traçado para obtenção do modelo final obtido.

Os resultados dos procedimentos utilizados para obtenção dos modelos para Latossolo Vermelho Distroférrico sob plantio direto foram registrados (Tabelas $4 \mathrm{a}$ 6).

No procedimento "backward elimination" gerado com os dados provindos do Latossolo Vermelho Distroférrico sob plantio direto (Tabela 4), o valor do coeficiente de determinação quando todas as variáveis são consideradas (passo 0) é de 0,49660619 .

Nos passos seguintes, as variáveis ARG, PT e MO são excluídas, apresentando um nível de significância acima de $10 \%$ de probabilidade.

Portanto, as variáveis DS e UG foram as únicas que contribuíram significativamente para explicar a relação entre RP e as variáveis independentes. 
Tabela 4. Procedimento "Backward elimination" para variável dependente (Y) resistência do solo à penetração (RP) de dados provindos do Latossolo Vermelho Distroférrico sob plantio direto

\begin{tabular}{|c|c|c|c|}
\hline Passo $0\left(\mathrm{R}^{2}=0,49660619\right)$ & Todas as variáveis consideradas & & \\
\hline Variáveis & Estimativa de parâmetros & $\mathrm{F}$ & Prob $>F$ \\
\hline Intercepto & $-9,0380$ & 17,72 & 0,0001 \\
\hline DS & $-4,4389$ & 17,96 & 0,0001 \\
\hline UG & 10,9162 & 89,75 & 0,0001 \\
\hline MO & 0,0073 & 0,50 & 0,4791 \\
\hline PT & 0,5057 & 0,17 & 0,6846 \\
\hline $\mathrm{ARG}$ & $-0,0007$ & 0,00 & 0,9559 \\
\hline Passo1 $\left(\mathrm{R}^{2}=0,49660095\right)$ & Remoção da variável ARG & & \\
\hline Intercepto & $-9,1105$ & 28,84 & 0,0001 \\
\hline DS & $-4,4388$ & 18,02 & 0,0001 \\
\hline UG & 10,9241 & 91,55 & 0,0001 \\
\hline MO & 0,0076 & 0,83 & 0,3642 \\
\hline PT & 0,5123 & 0,17 & 0,6789 \\
\hline Passo2 $\left(\mathrm{R}^{2}=0,49630792\right)$ & Remoção da variável PT & & \\
\hline Intercepto & $-8,7069$ & 39,40 & 0,0001 \\
\hline DS & $-4,4759$ & 18,51 & 0,0001 \\
\hline UG & 10,8352 & 93,62 & 0,0001 \\
\hline MO & 0,0073 & 0,77 & 0,3810 \\
\hline Passo3 $\left(\mathrm{R}^{2}=0,49499831\right)$ & Remoção da variável MO & & \\
\hline Intercepto & $-8,9431$ & 43,23 & 0,0001 \\
\hline DS & $-4,3990$ & 18,02 & 0,0001 \\
\hline UG & 11,2421 & 121,76 & 0,0001 \\
\hline
\end{tabular}

*Todas as variáveis participantes do modelo são significativas ao nível de $0,1000 \%$ de probabilidade. RP resistência do solo à penetração; DS - densidade do solo; UG - umidade gravimétrica; MO - matéria orgânica; PT porosidade total; ARG - argila. 
Tabela 5. Procedimento "Forward selection" para variável dependente (Y) resistência do solo à penetração (RP) de dados provindos do Latossolo Vermelho Distroférrico sob plantio direto

\begin{tabular}{cccc}
\hline $\begin{array}{c}\text { Passo } 1\left(\mathrm{R}^{2}=0,46436051\right) \\
\text { Variáveis }\end{array}$ & Todas as variáveis consideradas & \\
intercepto & Estimativa de parâmetros & $\mathrm{F}$ & Prob $>\mathrm{F}$ \\
UG & $-13,0002$ & 170,72 & 0,0001 \\
\hline Passo 2 $\left(\mathrm{R}^{2}=0,49499831\right)$ & 13,7413 & 258,34 & 0,0001 \\
\hline intercepto & & & \\
DS & $-8,9431$ & 43,23 & 0,0001 \\
UG & $-4,3989$ & 18,02 & 0,0001 \\
Passo3 (R $\left.{ }^{2}=0,49630792\right)$ & 11,2422 & 121,76 & 0,0001 \\
\hline intercepto & & & \\
DS & $-8,7069$ & 39,40 & 0,0001 \\
UG & $-4,4759$ & 18,51 & 0,0001 \\
MO & 10,8352 & 93,62 & 0,0001
\end{tabular}

*Todas as variáveis participantes do modelo são significativas ao nível de $0,5000 \%$ de probabilidade. RP resistência do solo à penetração; DS - densidade do solo; UG - umidade gravimétrica; MO - matéria orgânica; PT porosidade total; ARG - argila.

Tabela 6. Procedimento "Stepwise selection" para variável dependente (Y) resistência do solo à penetração (RP)de dados provindos do Latossolo Vermelho Distroférrico sob plantio direto

\begin{tabular}{cccc}
\hline $\begin{array}{c}\text { Passo } 1\left(\mathrm{R}^{2}=0,46436051\right) \\
\text { Variáveis }\end{array}$ & $\begin{array}{c}\text { Todas as variáveis consideradas } \\
\text { Estimativa de parâmetros }\end{array}$ & $\mathrm{F}$ & Prob>F \\
\hline Intercepto & $-13,0002$ & 170,72 & 0,0001 \\
$\mathrm{UG}$ & 13,7413 & 258,34 & 0,0001 \\
\hline Passo 2 $\left(\mathrm{R}^{2}=0,49499831\right)$ & & & \\
Intercepto & $-8,9431$ & 43,23 & 0,0001 \\
DS & $-4,3989$ & 18,02 & 0,0001 \\
$\mathrm{UG}$ & 11,2422 & 121,76 & 0,0001 \\
\hline
\end{tabular}

*Todas as variáveis participantes do modelo são significativas ao nível de $0,1500 \%$ de probabilidade. RP resistência do solo à penetração; DS - densidade do solo; UG - umidade gravimétrica; MO - matéria orgânica; PT porosidade total; ARG - argila. 
Do mesmo modo, Prado et al. (2002) constataram, em condições de campo, correlação próxima ao valor obtido neste trabalho $\left(\mathrm{r}=0,45^{\mathrm{ns}}\right)$ entre a resistência do solo à penetração e a densidade do solo na camada de 0-0,4 $\mathrm{m}$ de um Latossolo Vermelho eutroférrico sob cultivo intensivo e após cinco anos de pousio.

Observa-se que procedimentos "forward" e "stepwise" igualaram-se na obtenção dos modelos finais (Tabelas 5 e 6), tendo como selecionadas no modelo final as variáveis $\mathrm{DS}$ e UG, considerando a prob $<\mathrm{F}$ abaixo de $5 \%$.

Os três procedimentos utilizados, apesar de seguirem caminhos distintos, levaram a um mesmo resultado para explicar a relação entre as variáveis estudadas, com a seleção das variáveis DS e UG. Portanto, para esse solo, qualquer um dos três procedimentos pode ser utilizado, concordando com a afirmação de Draper \& Smith (1966), que apesar de haver vários procedimentos para seleção de um melhor modelo matemático para explicar a relação entre as variáveis estudadas, as soluções encontradas são muitas vezes iguais.

\section{CONCLUSÕES}

O procedimento "Stepwise" foi o mais adequado para a obtenção dos modelos finais para estimativa da qualidade física do Latossolo Vermelho Eutroférrico sob cultura perene, com o seguinte modelo final obtido: $\mathrm{RP}=1,5288-6,9237 \mathrm{DS}+5,9071 \mathrm{UG}+0,00115$ MO-0,00242ARG $\left(\mathrm{R}^{2}=0,60\right)$.

Os três procedimentos (stepwise, forward e backward) foram adequados na obtenção dos modelos finais para o Latossolo Vermelho Distroférrico sob plantio direto, com o modelo final obtido: $\mathrm{RP}=-9,431-4,3990 \mathrm{DS}+11,2421 \mathrm{UG}$ $\left(\mathrm{R}^{2}=0,49\right)$.

\section{REFERÊNCIAS BIBLIOGRÁFICAS}

AGUIAR, M. I. 2008. Qualidade física do solo em sistemas agroflorestais, 2008. (Dissertação de mestrado) Universidade Federal de Viçosa, Viçosa-MG, 91p.

ALBUQUERQUE, J. A.; SANGOI, L.; ENDER, M. 2001. Efeitos da integração lavoura-pecuária nas propriedades físicas do solo e características da cultura do milho. Revista Brasileira de Ciência do Solo, Viçosa-MG, v.25, p.717-723

ALMEIDA, C. X.; CENTURION, J. F.; FREDDI, O. S.; JORGE, R. F.; BARBOSA, J. C. 2008. Funções de pedotransferência para curva de resistência à penetração. Revista Brasileira de Ciência do Solo, ViçosaMG, v.32, p.2235-2243

ARAÚJO, R.; GOEDERT, W. J.; LACERDA, M. P. C. 2007. Qualidade de um solo sob diferentes usos e sob cerrado nativo. Revista Brasileira de Ciência do Solo, Viçosa-MG, v.31, p.1099-1108

BUSSCHER, W.J. 1990. Adjustment of flattipped penetrometer resistance data to a common water content. Trans. Am. Soc. Agron. Eng., v.33, p.519-524

BUSSCHER, W.J.; BAUER, P.J.; CAMP, C.R. \& SOJKA, R.E. 1997. Correction of cone index water content differences in a Coastal Plain soil. Soil Till. Res., v.43, p.205-217

DEXTER, A. R.; CZYZ, E. A.; GATE, O. P. 2007. Um método para predição de resistência à penetração do solo. Res. de preparo do solo, v.93, p.412-419

DIAS, F. L. F., MAZZA, J A., MATSUOKA, S., PERECIN, D., MAULE, R. F. 1997. Relação entre produtividade, clima, solos e variedades de cana-de-açúcar na região noroeste do Estado de São Paulo. Revista Brasileira de Ciência do Solo, Viçosa-MG, v.23, p.627-34 
DRAPER, N.R.; H. SMITH. 1966. Applied regression Analysis. New York

EFROYMSON, M. A. 1960. Multiple regression analysis. Mathematical methods for digital computers. Baston A and Welf, H.S, (eds), Wiley, New York

EMBRAPA. 2013. Sistema brasileiro de classificação de solos. 3ed. Brasília: EMBRAPA, 353p.

FIDALSKI, J.; TORMENA, C.A. 2007. Homogeneidade da qualidade física do solo nas entrelinhas de um pomar de laranjeira com sistemas de manejo da vegetação na entrelinha. Revista Brasileira de Ciência do Solo, ViçosaMG, v.31, n.4, p.637-645

HOCKING, R.R. 1976. The analysis and selection of variables in linear regression. Journal of the Biometrics Society. v. 32, n.1

IMHOFF, S.; SILVA, A. P. da; TORMENA, C. A. 2000. Aplicações da curva de resistência no controle da qualidade de um solo sob pastagem. Pesquisa Agropecuária Brasileira, Brasília, v. 35, n. 7, p. 1493-1500, Jul.

LANZANOVA, M. E.; NICOLOSO, R. S.; LOVATO, T.; ELTZ, F. L. F.; AMADO, T. J. C.; REINERT, D. J. 2007. Atributos físicos do solo em sistema de integração lavoura-pecuária sob plantio direto. Revista Brasileira de Ciência do Solo, Viçosa-MG, v.31, p.1131-1140

LIMA, C. L. R.; REICHERT, J. M.; REINERT, D. J.; SUZUKI, L. E. A. S.; DALBIANCO, L. 2007. Densidade crítica ao crescimento de plantas considerando água disponível e resistência à penetração de um Argissolo Vermelho distrófico arênico. Ciência Rural, v.37, p.1166-1169

PRADO, R.M.; ROQUE, C.G. \& SOUZA, Z.M. 2002. Sistemas de manejo e resistência do solo à penetração e densidade do solo de um Latossolo Vermelho eutroférrico em cultivo intensivo e pousio. Pesquisa Agropecuária Brasileira, v.37, p.17951801

RIBON, A. A.; TAVARES FILHO, J. 2004. Model propositions for the estimation of the physical quality of a Yellow Red Latosol (Oxisol) under pasture. Braz. Arch. Biol. Technol., v.47, p.25-31

RIBON, A. A.; TAVARES FILHO, J.; PERRI NETO, J. 2001. Proposição de modelos para estimativa da qualidade física de um Latossolo VermelhoAmarelo sob pastagem. In: XXVIII Congresso Brasileiro de Ciência do Solo, 2001, Londrina

SÁ, M. A. C.; SANTOS JÚNIOR, J. D. G. 2005. Compactação do solo: consequências para o crescimento vegetal. Planaltina: Embrapa Cerrados, 26p. Documentos, 136

SILVA, A. P.; KAY, B. D. 1997. A estimativa do intervalo hídrico ótimo de solos de propriedades e gestão. Sci solo. Soc. Sou. J., v.61, p.877-883

SINGER, M.; EWING, S. 2000. Soil quality. In: SUMNER, M. E. (ed.). Handbook of soil science. Boca Raton: CRC Press, p.271-298.

STEFANOSKI, D. C.; SANTOS, G. G.; MARCHÃO, R. L.; PETTER, F. A.; PACHECO, L. P. 2013. Uso e manejo do solo e seus impactos sobre a qualidade física. Revista Brasileira de Engenharia Agrícola e Ambiental, v.17, v.12, p.1301-1309

STONE, L. F.; FERREIRA, E. P. de B.; DIDONET, A. D.; HEINEMANN, A. B.; OLIVEIRA, J. P. de. 2013. Correlação entre a produtividade do feijoeiro no sistema de produção orgânica e atributos do solo. Revista Brasileira de Engenharia Agrícola e Ambiental, v.17, n.1, p.19-35

TAVARES FILHO, J.; FELTAN, C. T. M.; OLIVEIRA, J. F. de; ALMEIDA, E. de; GUIMARÃES, M. de F. 2012. Atributos de solo determinantes para estimativa do 
índice de estabilidade de agregados. Pesquisa Agropecuária Brasileira, v.47, n.3, p.436-441

TO, J.; KAY, B. D. 2005. Variação da resistência à penetração com as propriedades do solo: A contribuição da tensão efetiva e implicações para funções de pedotransferência. Geoderma, v.126, p.261-276

Recebido em: 16/11/2013 Aceito para publicação em: 28/2/2014 